\title{
The Impact of Arterial Collateralization on Outcome after Intra-Arterial Therapy for Acute Ischemic Stroke
}

\author{
S. Seeta Ramaiah, L. Churilov, P. Mitchell, R. Dowling, and B. Yan
}

\begin{abstract}
BACKGROUND AND PURPOSE: Although intra-arterial therapy for acute ischemic stroke is associated with superior recanalization rates, improved clinical outcomes are inconsistently observed following successful recanalization. There is emerging concern that unfavorable arterial collateralization, though unproven, predetermines poor outcome. We hypothesized that poor leptomeningeal collateralization, assessed by preprocedural CTA, is associated with poor outcome in patients with acute ischemic stroke undergoing intra-arterial therapy.
\end{abstract}

MATERIALS AND METHODS: We retrospectively analyzed patients with acute ischemic stroke with intracranial ICA and/or MCA occlusions who received intra-arterial therapy. The collaterals were graded on CTA. Univariate and multivariate analyses were used to investigate the association between the dichotomized leptomeningeal collateral score and functional outcomes at 3-months mRS $\leq 2$, mortality, and intracranial hemorrhages.

RESULTS: Eighty-seven patients were included. The median age was 66 years (interquartile range, 54-76 years) and the median NIHSS score at admission was 18 (interquartile range, 14-20). The leptomeningeal collateral score 3 was found to have significant association with the good functional outcome at 3 months: $\mathrm{OR}=3.13 ; 95 \% \mathrm{Cl}, 1.25-7.825 ; P=.016$. This association remained significant when adjusted for the use of IV tissue plasminogen activator: alone, $\mathrm{OR}=2.998 ; 95 \% \mathrm{Cl}, 1.154-7.786 ; P=.024$; and for IV tissue plasminogen activator and other confounders (age, baseline NIHSS score, and Thrombolysis in Cerebral Infarction grades), OR = 2.985; 95\% Cl, 1.027-8.673; $P=.045$.

CONCLUSIONS: We found that poor arterial collateralization, defined as a collateral score of $<3$, was associated with poor outcome, after adjustment for recanalization success. We recommend that future studies include collateral scores as one of the predictors of functional outcome.

ABBREVIATIONS: IAT = intra-arterial therapy; IQR = interquartile range

ntravenous tissue plasminogen activator is the only proved reperfusion therapy for acute ischemic stroke. However, a narrow therapeutic time window ( $<4.5$ hours) limits its use because the clinical effectiveness is critically time-dependent. ${ }^{1-3}$ In addition, recanalization rates with IV-tPA are low in the setting of large-artery occlusion, (eg, ICA occlusion $<10 \%$ ). ${ }^{4-6}$ Intra-arterial therapy (IAT) has higher recanalization rates than intravenous thrombolysis, but this result has not been matched by concordant improvement in clinical outcomes. ${ }^{7-9}$ Two recent randomized trials comparing IAT with IV-tPA, the Interventional

Received July 25, 2013; accepted August 26.

From the Melbourne Brain Centre (S.S.R., B.Y.) and Neurointervention Service (P.M., R.D., B.Y.), Department of Radiology, Royal Melbourne Hospital, Parkville, Victoria, Australia; and Department of Mathematics and Statistics (L.C.), and National Stroke Research Institute (L.C.), Florey Neuroscience Institutes, Austin Health, University of Melbourne, Parkville, Victoria, Australia.

Please address correspondence to Bernard Yan, MD, Royal Melbourne Hospital, 300 Grattan St, Parkville, Victoria 3050, Australia; e-mail: Bernard.yan@mh.org.au

http://dx.doi.org/10.3174/ajnr.A3862
Management of Stroke III trial and the Local versus Systemic Thrombolysis for Acute Ischemic Stroke trial, did not demonstrate superiority. ${ }^{10,11}$

Inadequate arterial collateralization is a possible mechanism to explain the mismatch between recanalization success and clinical outcome, apart from the presence of an already infarcted ischemic core and an incomplete microcirculatory reperfusion after focal cerebral ischemia. ${ }^{12,13}$ A favorable arterial collateralization as determined by a robust leptomeningeal anastomoses profile may enhance recanalization, improve downstream reperfusion, reduce the extent of infarct core and ischemic lesion growth, decrease hemorrhagic transformation, and improve outcome postrevascularization. ${ }^{14-16}$

The leptomeningeal collateral scoring system based on CTA correlates with clinical outcome. ${ }^{17-21}$ However, its role in IAT is unclear. We hypothesized that a poor leptomeningeal CTA score predicts clinical futility in patients undergoing IAT independent of recanalization status. 

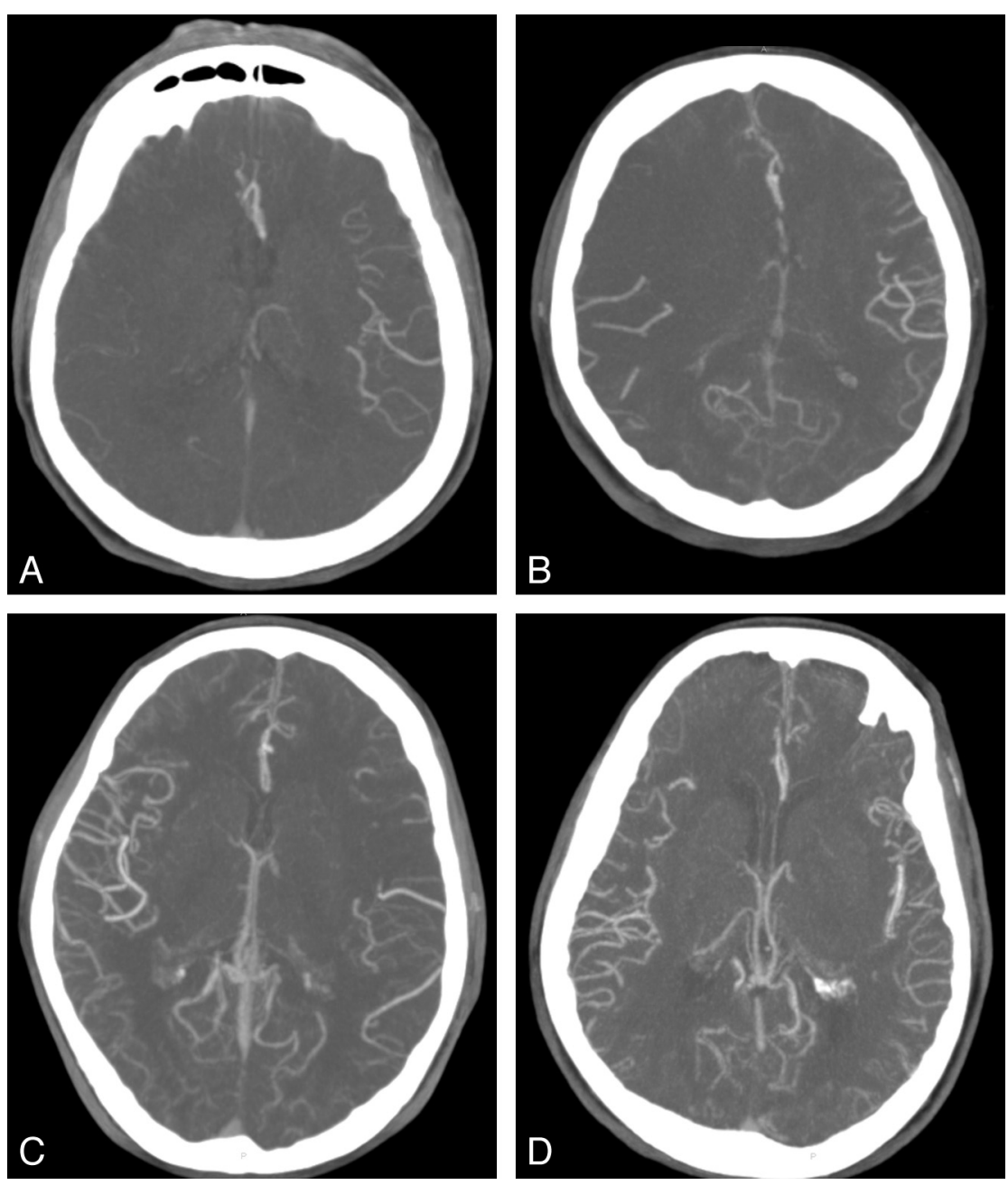

FIG 1. Leptomeningeal collateral CTA scores; $A$, score $=0 ; B$, score $=1 ; C$, score $=2 ; D$, score $=3$.

\section{MATERIALS AND METHODS}

\section{Study Design and Patient Cohort}

This was a single-center retrospective review of 104 patients with acute ischemic stroke with ICA and/or MCA occlusion who received IAT at the Royal Melbourne Hospital from January 2008 to March 2013. The local research and ethics committee granted the study approval.

Clinical parameters, identified through computerized data bases, were prospectively collected. The following parameters were recorded in a specific data bank: 1) demographics (age, sex); 2) medical history and risk factors such as hypertension (previous clinical diagnosis or regular treatment with antihypertensive medication), diabetes mellitus (previous diagnosis or current treatment with insulin or oral hypoglycemic medication), hypercholesterolemia (previous diagnosis or current treatment with lipid-lowering medication), atrial fibrillation (previous diagnosis or evident on admission), coronary artery disease, history of previous stroke or TIA; 3 ) "time from onset of symptoms to CTA" (defined from the time of symptom onset or from the time when the patient was last seen neurologically well, to the time of CTA), "time from onset of symptoms to recanalization" (defined from the time of symptom onset, or from the time when the patient was last seen neurologically well, to the time of intervention with any form of IAT), administration of IV-tPA before endovascular therapy, administration of intra-arterial tPA; and 4) clinical outcome variables: 3-month mRS score, intracranial hemorrhage, and 1-month mortality. The baseline severity of neurologic deficits was graded on admission according to the NIHSS.

\section{CTA and DSA Analysis}

CTAs of all patients were reviewed by 3 neurointerventionists (B.Y., P.M., and R.D.), with consensus opinion reached on collateral supply. The reviewers were blinded to all clinical information during the consensus collateral grading. The CTA source images (20-mm, axial) were edited with MPR technique for assessment of leptomeningeal collaterals based on distribution of vessels at the Sylvian fissure and the leptomeningeal convexity. The MPR images were assessed with an MIP technique at $32 \mathrm{~mm}$ to evaluate the leptomeningeal collaterals. Collateral statuses were divided into 4 categories (Fig 1): score $0=$ absence of contrast reaching the cortical surface of the affected hemisphere; score $1=$ contrast reaching the cortical surface but not the Sylvian fissure; score 2 = contrast reaching the Sylvian fissure but opacifying $<50 \%$ of hemisphere; score $3=$ contrast reaching the Sylvian fissure and opacifying $>50 \%$ of the hemisphere.

The Thrombolysis in Cerebral Infarction system was used to grade recanalization success. ${ }^{22}$ TICI grade 0 is no perfusion and no antegrade flow beyond the point of occlusion. TICI grade 1 is penetration with minimal perfusion. The contrast material passes beyond the area of obstruction but fails to opacify the entire cerebral bed distal to the obstruction for the duration of the angiographic run. TICI grade 2 is partial perfusion. The contrast material passes beyond the obstruction and opacifies the arterial bed distal to the obstruction. However, the rate of entry of contrast into the vessel distal to the obstruction or its rate of clearance from the distal bed or both are perceptibly slower than its entry into and/or clearance from comparable areas not perfused by the previously occluded vessel (eg, the opposite cerebral artery or the arterial bed proximal to the obstruction). In TICI grade 2a, only partial filling (less than two-thirds) of the entire vascular territory is visualized; in TICI grade $2 \mathrm{~b}$, complete filling of all of the expected vascular territory is visualized, but the filling is slower than normal. TICI grade 3 is complete perfusion; antegrade flow into the bed distal to the obstruction occurs as promptly as that into the obstruction and clearance.

\section{Statistics}

Statistical analysis was performed by using the STATA, Version 12 IC statistics package (StataCorp, College Station, Texas). We in- 
cluded the following baseline clinical variables: age, sex, comorbidities (history of hypertension, diabetes, atrial fibrillation, coronary artery disease, TIA, or stroke), baseline NIHSS score, IVtPA, intra-arterial tissue plasminogen activator, onset time to CTA, and onset time to recanalization. Continuous variables were reported as median \pm interquartile range (IQR). Categoric variables were reported as proportions. $P$ values were reported by using the Fisher exact test for categoric variables and the WilcoxonMann-Whitney rank sum test for continuous variables (age, NIHSS score, and time-to-event data). Effect sizes were reported as odds ratios for categoric variables and Hodges-Lehman median differences for continuous variables (age, NIHSS score, and timeto-event data). Receiver operating characteristic analysis curves were used to determine the collateral score threshold with functional outcome (3-month mRS score). Univariate and multivariate analyses were used to investigate the association between the dichotomized leptomeningeal collateral score with functional outcomes, mortality, and intracranial hemorrhages. Given the strong clinical association with clinical outcomes, we planned a priori to include the following independent variables in the model: age, baseline NIHSS score, TICI grade, and IV-tPA. A 2 -sided $P$ value $<.05$ was considered significant.

\section{RESULTS}

Of the initial 104 patients with acute ischemic stroke with ICA and/or MCA occlusion who underwent IAT, CTA was not available for 14 patients before IAT. Three patients had poor image quality on CTA, and these patients were excluded. Eighty-seven patients with both adequate CTA and angiographic data with clinical outcome (mRS) at 90 days available were entered into the leptomeningeal collateral assessment. The baseline characteristics did not differ in a statistically significant manner between the patients with and without CTA (Table 1). In the CTA group, the median age was 66 years (IQR, 54-76 years). Fifty (57\%) patients were men. The median NIHSS score at admission was 18 (IQR, 14-20). Forty $(46 \%)$ subjects were treated with IVtPA; 13 subjects $(15 \%)$ received intraarterial tPA. Sixty-five patients (75\%) were treated with the Solitaire device (Covidien, Irvine, California), 10 (12\%) patients were treated with the Merci retriever (Concentric Medical, Mountain View, California), and 3 (3\%) were treated with the Penumbra System (Penumbra, Alameda, California).

\section{Leptomeningeal Collaterals}

According to the leptomeningeal score, 34 subjects (39\%) were graded as three, 30 subjects (35\%) were graded as two, 12 subjects (14\%) were graded as 1 , and 11 subjects $(13 \%)$ were graded as zero. Receiver operating characteristic analysis

Note:-IA-tPA indicates intra-arterial tissue plasminogen activator.

a $P$ values reported using the Fisher exact test for categoric variables and the Wilcoxon-Mann-Whitney rank sum test for continuous variables (age, NIHSS score, and time-to-event data).

${ }^{b}$ Symptom onset or from the time when the patient was last seen neurologically well to the time of CT.

'Time from symptom onset or from the time when the patient was last seen neurologically well to the time of intervention with any form of IAT.

Table 2: Comparison of patients for the dichotomized leptomeningeal collateral score

\begin{tabular}{|c|c|c|c|c|}
\hline Characteristics & Collateral Score $3(n=34)$ & Collateral Score 0-2 (n= 53) & Effect Size $^{a}(95 \% \mathrm{Cl})$ & $P$ Value $^{\mathrm{b}}$ \\
\hline \multicolumn{5}{|l|}{ Baseline characteristics } \\
\hline Male sex & $16(47 \%)$ & $34(64 \%)$ & $2.013(0.844-4.802)$ & .127 \\
\hline NIHSS score, median (IQR) & $15.5(11-20)$ & $18(14-22)$ & $2(0-5)$ & .116 \\
\hline Hypertension & $18(53 \%)$ & $22(42 \%)$ & $1.585(0.671-3.745)$ & .379 \\
\hline Diabetes & $3(9 \%)$ & $8(15 \%)$ & $0.544(0.146-2.067)$ & .517 \\
\hline Dyslipidemia & $10(29 \%)$ & $16(30 \%)$ & $0.964(0.381-2.442)$ & $<.999$ \\
\hline $\mathrm{AF}$ & $10(30 \%)$ & $19(36 \%)$ & $0.746(0.299-1.863)$ & .643 \\
\hline CAD & $6(18 \%)$ & $5(9 \%)$ & $2.057(0.606-6.975)$ & .327 \\
\hline Previous stroke or TIA & $4(12 \%)$ & $9(17 \%)$ & $0.652(0.195-2.204)$ & .556 \\
\hline \multicolumn{5}{|l|}{ Process-of-care characteristics } \\
\hline IV-tPA & $22(65 \%)$ & $18(34 \%)$ & 3.565 (1.455-8.729) & .008 \\
\hline IA-tPA & $2(6 \%)$ & $11(21 \%)$ & $0.239(0-1.042)$ & .070 \\
\hline Onset to $\mathrm{CTA}^{\mathrm{C}}$ (min) (median) (IQR) & $90(63-124)$ & $90(75-137)$ & $4(-12-24)$ & .651 \\
\hline Recanalization $^{d}$ (min) (median) (IQR) & $146(86.5-274)$ & $175(128.68-276.5)$ & $-32.5(-72-14)$ & .169 \\
\hline $\mathrm{TICl} 2 \mathrm{~b}-3$ & $25(74 \%)$ & $35(66 \%)$ & $1.429(0.552-3.696)$ & .488 \\
\hline
\end{tabular}

Note:-IA-tPA indicates intra-arterial tissue plasminogen activator; AF, atrial fibrillation; CAD, coronary artery disease.

a Effect sizes reported as odds ratios for categoric variables and Hodges-Lehman median differences for continuous variables (age, NIHSS score, and time-to-event data).

${ }^{b} P$ values reported using the Fisher exact test for categoric variables and the Wilcoxon-Mann-Whitney rank sum test for continuous variables (age, NIHSS score, and time-to-event data).

' Symptom onset or from the time when the patient was last seen neurologically well to the time of CTA.

${ }^{\mathrm{d}}$ Time from symptom onset or from the time when the patient was last seen neurologically well to the time of intervention with any form of intra-arterial therapy. 
demonstrated the suitability of the collateral score $\geq 3$ for determining good functional outcome of $\mathrm{mRS} \leq 2$ with an area under the curve of 0.6513 (sensitivity, 51.1\%; specificity, $73.8 \%)$. The leptomeningeal score was dichotomized into good (score $=3$ ) and poor (score $=0-2$ ) on the basis of the receiver operating characteristic analysis. A higher percentage of IV-tPA was administered for a collateral score of 3 than for a collateral score of $0-2,(P=.008)$. The remainder of the baseline clinical variables did not differ in a statistically significant way between the 2 groups (Table 2).

\section{Outcome}

Three-month outcome was favorable ( $\mathrm{mRS} 0-2)$ in 47 subjects (54\%), and the remaining 40 subjects (46\%) had mRS scores of 3-6 (Fig 2). Patients with a good collateral score of 3 had higher odds of good functional outcome than patients with a collateral score of $0-2(\mathrm{OR}=3.130 ; 95 \% \mathrm{CI}, 1.252-7.825 ; P=.016)$. This finding remained significant after adjustment for administration of IV-tPA (OR = 2.998; 95\% CI, 1.154-7.786; $P=.024)$. In the multivariate analysis, patients with a collateral score of 3 had significantly higher odds of good outcome at 3-month follow-up after adjustment for age, baseline NIHSS score, IV-tPA, and TICI grades (adjusted OR $=2.985 ; 95 \% \mathrm{CI}, 1.027-8.673 ; P=.045$ ) (Table 3).

\section{Survival}

Of the 13 deaths at 1 month, patients with poor collateral scores of 0-2 exhibited a trend toward a higher death rate than those with a collateral score of 3 ( 11 versus 2 ) (OR $=0.239$; 95\% CI, 0.049 1.153; $P=.070)$, though no statistically significant differences were found.

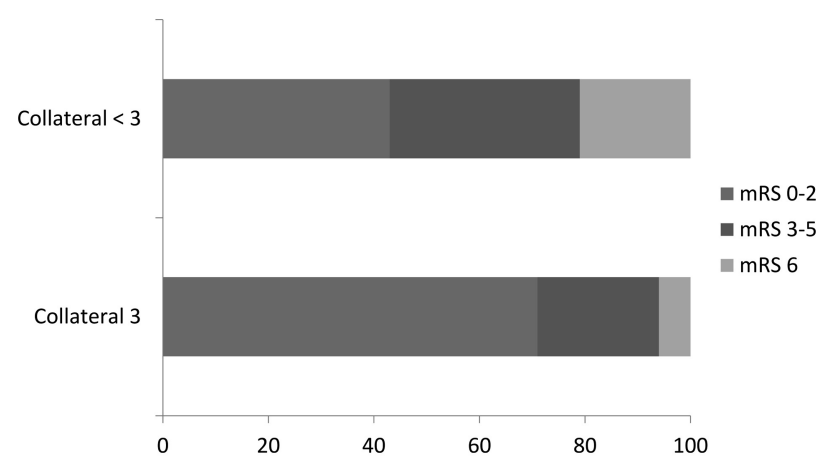

FIG 2. Distribution of mRS scores according to the leptomeningeal collateral scores.

\section{Intracranial Hemorrhage}

Although patients with a good collateral score of 3 had fewer episodes of intracranial hemorrhage than patients with a poor collateral score of $0-2$ (4 versus 13), no statistically significant difference was detected $(\mathrm{OR}=0.41 ; 95 \% \mathrm{CI}, 0.122-1.385 ; P=$ $.174)$.

\section{DISCUSSION}

We found that a favorable pattern of leptomeningeal collaterals, as measured by CTA on admission, was associated with improved functional outcomes at 3 months in a cohort of patients with acute ischemic stroke with ICA or proximal MCA occlusion. In a multivariate model, a good leptomeningeal collateral score remained independently associated with good clinical outcome after adjustment for age, baseline NIHSS score, IV-tPA, and recanalization status.

Various studies have used CTA to score collaterals status $^{14,17,19,20,23}$ with either CTA source images or by using MIP. Tan et $\mathrm{al}^{17}$ demonstrated that CTA-MIP was the best technique to quantify the degree of collateral circulation. In their retrospective analysis, they reported a good correlation between the CTA-based collateral score and the final infarct volume within 6 months. ${ }^{17}$ In another study, Miteff et $\mathrm{al}^{14}$ assessed retrograde filling of the MCA by 3 categories of collateral scoring and concluded that a good collateral status was one of the significant univariate predictors of favorable outcome. ${ }^{14}$ Menon et $\mathrm{al}^{21}$ included 138 patients (MCA-M1 and/or intracranial occlusion) in a retrospective single-center study and demonstrated good regional leptomeningeal anastomoses scores in $37.6 \%$ of patients, which correlated strongly with the size of the infarct core at baseline and were a strong independent predictor of final infarct and clinical outcome.

In a recent study, Souza et $\mathrm{al}^{23}$ found that CTA collaterals correlate with admission diffusion-weighted imaging infarct size and that a malignant collateral profile is highly specific for large admission DWI lesion size and poor functional outcome. In another study, Angermaier et $\mathrm{al}^{24}$ demonstrated that the CTA collateral grade was an independent predictor of final infarct volume in patients with stroke treated with endovascular therapy. On the other hand, Rosenthal et al ${ }^{18}$ reported that CTA collaterals had a positive impact on the outcomes of patients who did not achieve complete recanalization and had no impact in patients who were completely recanalized. Tan et $\mathrm{al}^{25}$ reported that good CTA collaterals correlated with improved outcomes in uni- but not in multivariate analyses. Our study reveals that a good CTA collateral score had a positive impact on the outcomes of patients in

Table 3: Univariate analysis of associations for outcomes for the dichotomized leptomeningeal score

\begin{tabular}{|c|c|c|c|c|c|c|c|c|}
\hline \multirow[b]{2}{*}{ Outcome } & \multirow{2}{*}{$\begin{array}{c}\text { Collateral } \\
\text { Score } 3 \\
(n=34)\end{array}$} & \multirow{2}{*}{$\begin{array}{c}\text { Collateral } \\
\text { Score } 0-2 \\
(n=53)\end{array}$} & \multicolumn{6}{|c|}{ ORs (95\% Cls) } \\
\hline & & & Unadjusted & $\begin{array}{c}P \\
\text { Value }^{\mathrm{a}}\end{array}$ & $\begin{array}{l}\text { Adjusted for } \\
\text { IV-tPA }\end{array}$ & $\begin{array}{c}P \\
\text { Value }^{\text {b }}\end{array}$ & Adjusted $^{c}$ & $\begin{array}{c}P \\
\text { Value }^{\text {b }}\end{array}$ \\
\hline $\begin{array}{l}\text { Clinical outcome mRS } \\
\leq 2 \text { at } 3 \text { months }\end{array}$ & $24(71 \%)$ & $23(43 \%)$ & $3.13(1.252-7.825)$ & .016 & 2.998 (1.154-7.786) & .024 & 2.985 (1.027-8.673) & .045 \\
\hline Intracranial hemorrhage & $4(12 \%)$ & $13(25 \%)$ & $0.41(0.122-1.385)$ & .174 & $0.5(0.141-1.770)$ & .283 & $0.510(0.141-1.850)$ & .306 \\
\hline Mortality at 1 month & $2(6 \%)$ & $11(21 \%)$ & 0.239 (0.049-1.153) & .070 & $0.21(0.041-1.073)$ & .061 & $0.215(0.036-1.284)$ & .092 \\
\hline
\end{tabular}

\footnotetext{
${ }^{a}$ Fisher exact test.

${ }^{\mathrm{b}}$ Logistic regression.

${ }^{c}$ Adjusted for age, baseline NIHSS score, IV-tPA, and TICI grades.
} 
both uni- and multivariate analyses independent of recanalization status.

The system of leptomeningeal anastomoses is anastomotic connections between distal branches of the cerebral arteries on the surface of the brain that permit blood flow from the territory of an unobstructed artery into the territory of an occluded artery and constitute the secondary network of cerebral collateral circulation apart from the circle of Willis. ${ }^{26}$ Although the criterion standard for the assessment of leptomeningeal anastomoses is conventional DSA, the extent of leptomeningeal anastomoses formation seen on conventional DSA and with clinical outcome correlates well with collateral blood flow as assessed by CTA. ${ }^{15,27,28}$ CTA is quicker, simpler, and noninvasive and uses IV-administered contrast to visualize the extent of leptomeningeal anastomoses with intra- and extracranial vasculature. ${ }^{19,21}$

There is currently no consensus on what the presence or absence of collateral circulation means with respect to treatment choices for patients with acute ischemic stroke. Some authors believe a minority of patients with robust leptomeningeal collateral circulation will have minimal damage and experience excellent recovery without recanalization. ${ }^{29}$ On the other hand, some neurointerventionalists may want to try more aggressive IAT because there may be salvageable tissue in patients with good collateral vessel formation. Given the risks inherent in the therapeutic procedure and the clinical risk of futile recanalization, ${ }^{30}$ practitioners need a way to consistently select patients whom IAT is likely to benefit rather than harm. Given the significance of leptomeningeal collaterals, they should be taken into account with other validated scoring systems, namely the Houston Intra-Arterial Therapy ${ }^{30}$ and the Totaled Heath Risks in Vascular Events score, ${ }^{31}$ in patient selection for IAT.

Our study has some limitations. First, selection bias is unavoidable in a retrospective single-center study. We sought to minimize reading bias by blinding the observers to clinical data and consensus achieved for the collateral grading. The CTA collateral scoring system was modified from Tan et al. ${ }^{17}$ Although our collateral grading derived from a consensus by 3 experienced neurointerventionists, the utility and value of the score requires further validation in a larger study before collateral scores are used as a primary means of selecting patients for revascularization therapy. Potential biases may have influenced the selection of cases for IAT on the basis of the CTA and clinical data.

\section{CONCLUSIONS}

Evaluation of the leptomeningeal collateral blood supply before IAT for anterior circulation intracranial arterial occlusion stroke (ICA and/or MCA) with a collateral score based on CTA-MIP reconstructions is independently associated with functional outcome at 3 months. We believe that leptomeningeal collateral assessment should be included in future trials investigating the clinical efficacy of IAT.

\section{REFERENCES}

1. The National Institute of Neurological Disorders and Stroke rt-PA Stroke Study Group. Tissue plasminogen activator for acute ischemic stroke. $N$ Engl J Med 1995;333:1581-87

2. Wahlgren N, Ahmed N, Eriksson N, et al. Multivariable analysis of outcome predictors and adjustment of main outcome results to baseline data profile in randomized controlled trials Safe Implementation of Thrombolysis in Stroke-Monitoring Study (SITSMOST). Stroke 2008;39:3316-22

3. Lees KR, Bluhmki E, von Kummer R, et al. Time to treatment with intravenous alteplase and outcome in stroke: an updated pooled analysis of ECASS, ATLANTIS, NINDS, and EPITHET trials. Lancet 2010;375:1695-703

4. Bhatia R, Hill MD, Shobha N, et al. Low rates of acute recanalization with intravenous recombinant tissue plasminogen activator in ischemic stroke: real-world experience and a call for action. Stroke 2010;41:2254-58

5. Christou I, Felberg RA, Demchuk AM, et al. Intravenous tissue plasminogen activator and flow improvement in acute ischemic stroke patients with internal carotid artery occlusion. J Neuroimaging 2002;12:119-23

6. Zangerle A, Kiechl S, Spiegel M, et al. Recanalization after thrombolysis in stroke patients: predictors and prognostic implications. Neurology 2007;68:39-44

7. Meyers PM, Schumacher HC, Connolly ES Jr, et al. Current status of endovascular stroke treatment. Circulation 2011;123:2591-601

8. Smith WS, Sung G, Saver J, et al. Mechanical thrombectomy for acute ischemic stroke: final results of the Multi MERCI trial. Stroke 2008;39:1205-12

9. Clark W, Lutsep H, Barnwell S, et al. The Penumbra Pivotal Stroke trial: safety and effectiveness of a new generation of mechanical devices for clot removal in intracranial large vessel occlusive disease. Stroke 2009;40:2761-68

10. Broderick JP, Palesch YY, Demchuk AM, et al, for the Interventional Management of Stroke (IMS) III Investigators. Endovascular therapy after intravenous t-PA versus t-PA alone for stroke. $N \mathrm{Engl}$ J Med 2013;368:893-903

11. Ciccone A, Valvassori L, Nichelatti M, et al. Endovascular treatment for acute ischemic stroke. N Engl J Med 2013;368:904-13

12. Chimowitz MI. Endovascular treatment for acute ischemic stroke: still unproven. N Engl J Med 2013;368:954-55

13. Dalkara T, Arsava EM. Can restoring incomplete microcirculatory reperfusion improve stroke outcome after thrombolysis? J Cereb Blood Flow Metab 2012;32:2091-209

14. Miteff F, Levi CR, Bateman GA, et al. The independent predictive utility of computed tomography angiographic collateral status in acute ischaemic stroke. Brain 2009;132(pt 8):2231-2238

15. Bang OY, Saver JL, Buck BH, et al, for the UCLA Collateral Investigators. Impact of collateral flow on tissue fate in acute ischaemic stroke. J Neurol Neurosurg Psychiatry 2007;79:625-29

16. Bang OY, Saver JL, Kim SJ, et al. Collateral flow predicts response to endovascular therapy for acute ischemic stroke. Stroke 2011;42: 693-99

17. Tan JC, Dillon WP, Liu S, et al. Systematic comparison of perfusion-CT and CT-angiography in acute stroke patients. Ann Neurol 2007;61:533-43

18. Rosenthal ES, Schwamm LH, Roccatagliata L, et al. Role of recanalization in acute stroke outcome: rationale for a CT angiogram-based "benefit of recanalization" model. AJNR Am J Neuroradiol 2008;29:1471-75

19. Maas MB, Lev MH, Ay H, et al. Collateral vessels on CT angiography predict outcome in acute ischemic stroke. Stroke 2009;40:3001-05

20. Lima FO, Furie KL, Silva GS, et al. The pattern of leptomeningeal collaterals on CT angiography is a strong predictor of long-term functional outcome in stroke patients with large vessel intracranial occlusion. Stroke 2010;41:2316-22

21. Menon BK, Smith EE, Modi J, et al. Regional leptomeningeal score on CT angiography predicts clinical and imaging outcomes in patients with acute anterior circulation occlusions. AJNR Am J Neuroradiol 2011;32:1640-45

22. Higashida RT, Anthony J Furlan, et al. Trial design and reporting standards for intraarterial cerebral thrombolysis for acute ischemic stroke. J Vasc Interv Radiol 2003;14(9 Pt 2):S493-94

23. Souza LC, Yoo AJ, Chaudhry ZA, et al. Malignant CTA collateral profile 
is highly specific for large admission DWI infarct core and poor outcome in acute stroke. AJNR Am J Neuroradiol 2012;33:1331-36

24. Angermaier A, Langner S, Kirsch M, et al. CT-angiographic collateralization predicts final infarct volume after intra-arterial thrombolysis for acute anterior circulation ischemic stroke. Cerebrovasc Dis 2011;31:177-84

25. Tan IY, Demchuk AM, Hopyan J, et al. CT angiography clot burden score and collateral score: correlation with clinical and radiologic outcomes in acute middle cerebral artery infarct. AJNR Am J Neuroradiol 2009;30:525-31

26. Liebeskind DS. Collaterals in acute stroke: beyond the clot. Neuroimaging Clin N Am 2005;15:553-573, x
27. Wildermuth S, Knauth M, Brandt $\mathrm{T}$, et al. Role of $\mathrm{CT}$ angiography in patient selection for thrombolytic therapy in acute hemispheric stroke. Stroke 1998;29:935-38

28. Liebeskind DS. Collateral circulation. Stroke 2003;34:2279-84

29. Tomsick TA, Khatri P, Jovin T, et al. Equipoise among recanalization strategies. Neurology 2010;74:1069-76

30. Hallevi Hen, Barreto AD, Liebeskind DS, et al. Identifying patients at high risk for poor outcome after intra-arterial therapy for acute ischemic stroke. Stroke 2009;40:1780-85

31. Flint AC, Kamel H, Rao VA, et al. Validation of the Totaled Heath Risks In Vascular Events (THRIVE) score for outcome prediction in endovascular stroke treatment. Int J Stroke 2014;9:32-39 